\title{
Impacto das mudanças vocais e auditivas na qualidade de vida de idosos ativos
}

\author{
Influence of voice and hearing changes \\ in the quality of life of active elderly individuals
}

\author{
Julia Santos Costa Chiossi ${ }^{1}$ \\ Francelise Pivetta Roque ${ }^{2}$ \\ Bárbara Niegia Garcia de Goulart ${ }^{3}$ \\ Brasilia Maria Chiari ${ }^{1}$
}

${ }^{1}$ Departamento de Fonoaudiologia, Universidade Federal de São Paulo. R. Botucatu 802, Vila Clementino. 04.023900 São Paulo SP Brasil. julia.chiossi@unifesp.br ${ }^{2}$ Polo Universitário de Nova Friburgo, Universidade Federal Fluminense. ${ }^{3}$ Departamento de Saúde e Comunicação Humana, Universidade Federal do Rio Grande do Sul.

\begin{abstract}
This article seeks to verify the self-rated impact of voice and hearing changes of active elderly individuals in their daily lives, and the influence of this self-rating on quality of life. A cross-sectional study was conducted with 72 elderly individuals of an Open University for Senior Citizens in the state of São Paulo. The questionnaires applied were HHIE-S; VHI and WHOQoL-Old. The Pearson correlation coefficient was used adopting a p-level significance value of $<0.05$. The impact of hearing difficulties on daily life was perceived by $45.8 \%$, and moderate or severe voice handicap by $9.7 \%$ of the elderly individuals. The self-rating of hearing impact on daily life was correlated with the voice handicap index. Quality of life was negatively affected by the increase in self-rating of hearing and voice difficulties in daily life. The sample profile is typical of successful aging with the acceptance of aging changes and consequently less impact on daily lives than expected. The findings suggest that there is an impact of voice and hearing handicap on quality of life, although it has revealed high indices, bolstering the characteristic of adaptation of the sample to aging. The results justify the need for improving actions of self-care and empowerment for the elderly.
\end{abstract}

Key words Daily activities, Hearing, Aging, Elderly, Quality of life, Voice
Resumo O objetivo do artigo é verificar a autopercepção de idosos ativos sobre o impacto de mudanças vocais e auditivas senescentes em sua vida diária, e a influência desta autopercepção na qualidade de vida. Realizou-se um estudo transversal com 72 idosos de uma Universidade Aberta à Terceira Idade paulistana. Aplicaram-se os questionários HHIE-S; IDV e WHOQoL-Old. Utilizou-se a correlação de Pearson adotando-se como significante o p-valor de 0,05. O impacto das dificuldades auditivas na vida diária foi percebido por $45,8 \%$, e a desvantagem vocal moderada ou severa, por 9,7\% dos idosos. A autopercepção de impacto auditivo na vida diária correlacionou-se com o indice de desvantagem vocal. A qualidade de vida foi influenciada negativamente pelo aumento da autopercepção de dificuldades auditivas e vocais no cotidiano. O perfil da amostra é típico da velhice bem-sucedida, com a aceitação das mudanças senescentes e, assim, com menor impacto dessas no seu cotidiano do que o hipotetizado. Os achados sugerem impacto da restrição da participação decorrente da voz e audição na qualidade de vida, embora essa tenha apresentado indices elevados, reforçando a característica de adaptação da amostra à senescência. Os resultados justificam a necessidade de ações de autocuidado e empoderamento para os idosos.

Palavras-chave Atividades cotidianas, Audição, Envelhecimento, Idoso, Qualidade de vida, Voz 


\section{Introdução}

Em face do envelhecimento mundial, também vivenciado no Brasil ${ }^{1}$, defendem-se ações que viabilizem o Envelhecimento Ativo da população, fundamentado no princípio de otimização das oportunidades de saúde, participação e segurança².

A manutenção da capacidade funcional é um aspecto essencial para o envelhecimento ativo ${ }^{3,4}$, sendo que as possibilidades de comunicação, seja a partir da compreensão das mensagens orais e escritas, ou pela possibilidade de expressar-se, são extremamente relevantes para a interação social, ação necessária à conservação da independência e autonomia.

Modificações no padrão e nas habilidades de comunicação ocorrem fisiologicamente no decorrer do envelhecimento ${ }^{5}$. Algumas delas podem afetar a qualidade de vida do idoso quando passam a interferir no seu convívio social ${ }^{4,6}$. Entre essas mudanças, a diminuição da audição, denominada presbiacusia, pode acarretar dificuldade em conversas em ambientes ruidosos ou com velocidade de fala aumentada ${ }^{7}$. As modificações na voz, constituintes da presbifonia, por sua vez, sejam decorrentes das mudanças no aparelho fonador ou por alterações no automonitoramento auditivo $^{6,8}$, podem ter grande influência em aspectos psicossociais do indivíduo idoso, ao interferir em seu funcionamento social ${ }^{9}$.

A percepção destes aspectos e sua influência na qualidade de vida, bem como a análise de sua presença em idosos ativos, são importantes para que ações mais efetivas de prevenção sejam planejadas para a promoção e manutenção do autocuidado, bem como para o diagnóstico e a intervenção precoces ${ }^{10}$.

Assim, o objetivo deste estudo é verificar a autopercepção do idoso ativo com relação ao impacto de mudanças vocais e auditivas senescentes em sua vida diária, e a influência desta autopercepção na sua qualidade de vida.

\section{Metodologia}

Trata-se de um estudo transversal, cuja amostra foi composta por idosos alfabetizados, frequentadores de uma Universidade Aberta à Terceira Idade (UATI) paulistana, participantes de um curso de idiomas (inglês ou espanhol) e/ou de um curso denominado "curricular", no qual são discutidos assuntos referentes à saúde, cultura e sociedade na velhice.

A UATI conta com cerca de 150 alunos por ano, com 50 anos ou mais, provenientes da Grande São Paulo, que procuram espontaneamente os cursos, seja por indicação, contato telefônico ou internet.

Todos os idosos, com 60 anos ou mais, participantes desses cursos eram elegíveis para o estudo, e foram recrutados por meio de visitas de uma das pesquisadoras à UATI nos dias letivos, entre outubro de 2010 e março de 2011. Foram critérios de exclusão a presença, nos idosos, de comprometimentos motores, visuais ou cognitivos, autodeclarados ou identificados pela pesquisadora durante a entrevista, que não permitissem que estes lessem e preenchessem os questionários, bem como a sua não autorização para participação no estudo. Para a avaliação do impacto da audição e da voz nas atividades diárias utilizaram-se o Hearing Handicap Inventory for the Elderly - Screening (HHIE-S ${ }^{11}$ e o Índice de Desvantagem Vocal (IDV) ${ }^{12}$, e para a avaliação da percepção da qualidade de vida utilizou-se o Questionário de Avaliação da Qualidade de Vida da Organização Mundial de Saúde para Idosos (World Health Organization Quality of Life - OLD - WHOQoL-OLD) ${ }^{13}$.

Além disso, características da população estudada como idade, sexo, estado civil, escolaridade, número de filhos, coabitação, atividades físicas e sociais das quais participava à época da coleta de dados, doenças, o curso de que participava na UATI foram verificados a partir de questionário com questões fechadas.

O HHIE-S é um questionário formado por 10 perguntas fechadas sobre as restrições de participação decorrentes de problemas auditivos, com respostas graduadas em "sim" (quatro pontos), "não" (nenhum ponto) e "às vezes" (dois pontos). Escores até 8 pontos indicam não haver percepção do handicap auditivo; de 10 a 24 pontos, uma percepção leve a moderada, e acima de 24, uma percepção significativa ${ }^{11}$. As perguntas estão divididas em dois domínios, social e emocional, com cinco perguntas cada um.

O IDV é composto por 30 itens referentes às restrições causadas por alterações vocais na vida diária; as respostas são graduadas de 0 a 4 , sendo 0 quando o indivíduo nunca compartilha da dificuldade descrita e 4 quando sempre ${ }^{12}$. Assim, os sujeitos foram classificados conforme o impacto na vida diária em desvantagem leve (até 40 pontos), desvantagem moderada (de 41 a 60 pontos) e desvantagem severa (acima de 60 pontos) ${ }^{14}$. O questionário também divide os fatores de restrição em emocionais, funcionais e orgânicos, com 10 itens cada um, dispostos em ordem aleatória. 
Para mensuração da qualidade de vida, utilizou-se o WHOQoL-OLD, desenvolvido pela Organização Mundial de Saúde (OMS) especificamente para idosos ${ }^{15}$, que consiste em 24 itens, pontuados de 1 a 5 , atribuídos a seis facetas: funcionamento sensorial; autonomia; atividades passadas, presentes e futuras; participação social; morte e morrer; e intimidade. O escore total varia de zero a 120 pontos, sendo que, quanto maior a pontuação, melhor a qualidade de vida.

Considerando a percepção da desvantagem auditiva a mais prevalente entre as questões estudadas, e assumindo a proporção de $25 \%$ de sujeitos com esse desfecho ${ }^{16-18}$, um erro de $10 \%$ e um intervalo de confiança de $95 \%$, o tamanho da amostra prevista foi de 72 pessoas, que correspondeu ao total de sujeitos dessa pesquisa.

Foi realizada análise de correlação entre os escores do IDV, HHIE-S e WHOQoL-OLD, e variáveis sociodemográficas, culturais e de saúde, por meio da Correlação de Spearman, considerando um nível de significância de 5\%.

Este estudo foi aprovado pelo Comitê de Ética e ao final foi proporcionada para todos os sujeitos que desejaram uma explicação sobre os escores obtidos nos testes, e para aqueles que apresentaram durante este estudo alguma queixa de dificuldade de audição ou de voz, lhes foram facilitadas as instruções sobre como serem aten- didos na triagem dos serviços de otorrinolaringologia ou do ambulatório de voz da instituição.

\section{Resultados}

As características sociodemográficas, culturais e de saúde dos idosos estão apresentadas na Tabela 1 . Os sujeitos praticavam atividades físicas em média $4 \pm 2$ dias por semana. Vinte e sete pessoas $(37,5 \%)$ referiram apenas uma doença, $10(13,9 \%)$ relataram duas doenças e $25(34,7 \%)$ afirmaram ter três ou mais doenças. As mais prevalentes foram hipertensão arterial sistêmica $(\mathrm{n}=35 ; 48,6 \%)$ e hipercolesterolemia $(\mathrm{n}=28$; $38,9 \%)$.

A Tabela 2 contém os valores de handicap auditivo, desvantagem vocal e qualidade de vida, obtidos.

Nenhum dos sujeitos incluídos na pesquisa era usuário de aparelho de amplificação sonora individual (AASI). Trinta e três sujeitos $(45,8 \%)$ perceberam a influência da audição na vida diária (HHIE-S > 8), e sete indivíduos $(9,7 \%)$ apresentaram desvantagem vocal moderada ou severa (IDV > 40). A verificação de correlação entre a autopercepção do impacto das modificações da voz e da audição na vida diária e a qualidade de vida pode ser observada na Tabela 3.

Tabela 1. Características sociodemográficas, culturais e de saúde dos idosos.

\begin{tabular}{|c|c|c|c|}
\hline Variáveis & Mulheres $(n=65)$ & Homens $(\mathbf{n}=7)$ & TOTAL $(\mathrm{n}=72)$ \\
\hline & \multicolumn{3}{|c|}{ Média (desvio padrão) } \\
\hline Idade (anos) & $68,5( \pm 6,2)$ & $68,3( \pm 6,9)$ & $68,5( \pm 6,2)$ \\
\hline Escolaridade (anos) & $11,9( \pm 3,5)$ & $14,1( \pm 1,7)$ & $12,1( \pm 3,4)$ \\
\hline Estado civil & \multicolumn{3}{|c|}{ N (\%) } \\
\hline Solteiro & $10(15,4)$ & $0(0,0)$ & $10(13,9)$ \\
\hline Casado & $18(27,7)$ & $5(71,4)$ & $23(31,9)$ \\
\hline Viúvo & $26(40,0)$ & $0(0,0)$ & $26(36,1)$ \\
\hline Divorciado & $11(16,9)$ & $2(28,6)$ & $13(18,1)$ \\
\hline \multicolumn{4}{|l|}{ Coabitação } \\
\hline Sozinho & $26(40,0)$ & $1(14,3)$ & $27(37,5)$ \\
\hline Cônjuge (com ou sem filhos) & $19(29,2)$ & $6(85,7)$ & $25(34,7)$ \\
\hline Só Filhos & $15(23,1)$ & $0(0,0)$ & $15(20,9)$ \\
\hline Outros & $5(7,7)$ & $0(0,0)$ & $5(6,9)$ \\
\hline \multicolumn{4}{|l|}{ Prática regular de atividade física } \\
\hline Não & $27(41,5)$ & $2(28,6)$ & $29(40,3)$ \\
\hline Sim & $38(58,5)$ & $5(71,4)$ & $43(59,7)$ \\
\hline \multicolumn{4}{|l|}{ Presença de doenças } \\
\hline Não & $9(13,9)$ & $1(14,3)$ & $10(13,9)$ \\
\hline Sim & $56(86,1)$ & $6(85,7)$ & $62(86,1)$ \\
\hline
\end{tabular}


$\mathrm{O}$ escore de qualidade de vida se relacionou à prática de exercícios físicos $(\mathrm{p}=0,011)$ e ao número de doenças crônicas $(\mathrm{p}<0,001)$. As demais variáveis sociodemográficas e culturais não apresentaram correlação com a autopercepção do impacto de modificações vocais e auditivas no cotidiano, nem com a qualidade de vida.

\section{Discussão}

A maior parte da amostra foi constituída de idosas jovens (60-69 anos), com alta escolaridade, viúvas ou casadas, característica comum a outros estudos realizados em Universidades da Terceira Idade ${ }^{19-21}$. Além disso, a maior parte dos idosos referiu apresentar pelo menos uma doença crônica, prevalência semelhante à média da população brasileira $^{1}$. Ao contrário de outras pesquisas ${ }^{19,21}$, a maior parte dos idosos estudados realiza alguma atividade física, possivelmente pela grande presença, nesta amostra, de sujeitos de cultura japonesa, com costume de prática diária e coletiva de exercícios físicos.

Tabela 2. Escores de percepção do impacto da e audição (HHIE-S) e voz (IDV) no cotidiano e de qualidade de vida (WHOQoL-OLD).

\begin{tabular}{lcrc}
\hline \multicolumn{1}{c}{ Variável } & Percentil 25 & Mediana & Percentil 75 \\
\hline HHIE-S & 2,0 & 8,0 & 16,0 \\
IDV & 3,0 & 8,5 & 20,0 \\
WHOQoL-OLD & 86,0 & 94,5 & 102,0 \\
\hline
\end{tabular}

Legenda: HHIE-S (Hearing Handicap Inventory for Elderly - Screening); IDV (Índice de Desvantagem Vocal); WHOQoL-OLD (Questionário de Avaliação da Qualidade de Vida da Organização Mundial de Saúde, para Idosos).

Tabela 3. Correlação entre a percepção de impactos vocais e auditivos no cotidiano e a qualidade de vida.

\begin{tabular}{lcc}
\hline \multicolumn{1}{c}{ Par de Variáveis } & \multicolumn{2}{c}{ Coeficiente de } \\
& Correlação $(\mathbf{r})$ & $\begin{array}{c}\text { Significância } \\
(\mathbf{p})\end{array}$ \\
\hline HHIE-S x IDV & $+0,445$ & $<0,001^{*}$ \\
WHOQoL-OLD x HHIE-S & $-0,341$ & $0,003^{*}$ \\
WHOQoL-OLD x IDV & $-0,297$ & $0,011^{*}$ \\
\hline
\end{tabular}

${ }^{*}$ : p estatisticamente significante, pela Análise de Correlação de Spearman.

Legenda: HHIE-S (Hearing Handicap Inventory for Elderly Screening); IDV (Îndice de Desvantagem Vocal); WHOQoL-OLD (Questionário de Avaliação da Qualidade de Vida da Organização Mundial de Saúde, para Idosos).
A proporção de idosos que percebem influência de alterações auditivas no cotidiano é bastante variada na literatura, com ocorrência entre 11 e $39 \%{ }^{16-18}$. Embora nossos dados tenham indicado uma maior proporção de sujeitos com esta percepção, o escore médio no teste reflete uma desvantagem leve, o que implica uma restrição da participação pouco significativa e, portanto, com pouca influência percebida nas atividades diárias. Por estar relacionada a fatores culturais e emocionais, a presbiacusia pode ser supervalorizada ou negada pelo idoso ${ }^{16}$; assim, há pessoas com deficiência mínima, mas dificuldades significantes, bem como pessoas com perda significante, sem impacto importante em seu cotidiano.

Apesar de sabida a influência do uso de aparelho de amplificação sonora individual na qualidade de vida dos idosos ${ }^{22}$, esta pesquisa não $o$ adotou como critério de inclusão ou exclusão, visto que o objetivo se relacionava à análise da funcionalidade comunicativa e sua relação com a qualidade de vida, mantendo-se, portanto, as características de voz e audição habituais dos participantes. Ainda assim, nos dados analisados, nenhum dos sujeitos era usuário de AASI, de maneira que este dado não foi incluído nas conclusões obtidas.

Os dados revelam ainda que a desvantagem auditiva percebida no domínio social foi mais importante do que a percebida no domínio emocional, apesar da relação, já comprovada em na literatura, entre a perda auditiva e as dificuldades emocionais ${ }^{6,23}$. Esse achado está em consonância com o de um estudo brasileiro, no qual também os aspectos sociais foram mais afetados do que os emocionais ${ }^{16}$.

Os achados do presente trabalho sugerem que a restrição da participação resultante das dificuldades auditivas influenciou na qualidade de vida dos idosos, dado que encontra consonância com estudos anteriores que versaram sobre o tema, ainda que com metodologia diversa ${ }^{24,25}$. Segundo alguns autores, os questionários de autoavaliação do impacto da audição nas atividades diárias, como o HHIE-S, têm uma relação mais forte com o nível de qualidade de vida que avaliação audiométrica ${ }^{26}$, uma vez que avaliam o estresse emocional e social do sujeito em suas relações com familiares e amigos.

Com menor proporção que para a audição, o número de idosos que percebeu a influência das mudanças vocais no cotidiano foi similar ao relatado na literatura ${ }^{27,28}$, sendo destacados os fatores orgânico-funcionais como mais relevantes nessa percepção, comparados aos aspectos emocionais, 
possivelmente porque a presbifonia é fisiologicamente decorrente de modificações anatômicas ${ }^{29}$, sendo este o domínio com mais impacto no cotidiano dos sujeitos.

Para os idosos que perceberam a influência de modificações vocais no cotidiano, verificou-se impacto na qualidade de vida, conforme já observado em outros estudos ${ }^{30-32}$.

Analisa-se, porém, que essa autopercepção pode apresentar índices menores do que a real presença de distúrbio vocal, quando verificado em avaliação específica ${ }^{28,33}$, possivelmente porque a importância dada à qualidade vocal na velhice é menor em comparação à importância da voz para o desempenho da atividade profissional, ou ainda por esta mudança não ser tão perceptível ao idoso como outras mudanças físicas senescentes ${ }^{30}$. Esse contraste reforça a necessidade de se avaliar não apenas a existência de alterações, mas se há restrição de participação dela resultante, visto que este aspecto também se correlaciona com a qualidade de vida.

A coocorrência de alterações de voz e audição já havia sido observada em estudo anterior ${ }^{6}$, no qual indivíduos com percepção da restrição de participação ocasionada por alterações auditivas, avaliada por meio do teste HHIE-S, apresentaram maior predisposição à presença de distúrbio vocal, possivelmente por precisarem aumentar a intensidade da fala para se escutarem. Ainda segundo os autores, pacientes com ambas as dificuldades tendem a sofrer de ansiedade e isolamento social, ocasionando maiores índices em escalas de depressão, com consequente interferência na sua qualidade de vida ${ }^{6}$.

A amostra tem características condizentes com o perfil típico de pessoas que vivenciam uma velhice bem-sucedida, conceito que traduz a aceitação das mudanças naturais da velhice, adaptação à presença das doenças comuns nessa fase da vida, adoção de hábitos saudáveis, e participação social $^{34}$. Isso pode justificar que o impacto percebido das mudanças auditivas e vocais no seu cotidiano seja menor em comparação ao que se esperaria caso essas modificações não fossem decorrentes do envelhecimento, ou caso não fossem bem adaptados a elas. Também pode explicar que o impacto emocional seja menor do que o social, no caso da audição, e orgânico-funcional, no caso da voz, afinal, embora eles demonstrem perceber mudanças no cotidiano decorrentes da comunicação senescente, estão bem adaptados a elas a ponto de os aspectos emocionais não serem tão afetados quanto os demais. Apesar da correlação entre o impacto percebido e a qualidade de vida, verifica-se que essa apresentou índices elevados, reforçando a característica de adaptação da amostra à senescência.

Considerando-se a possível interferência de outros fatores na qualidade de vida além dos que foram principal alvo deste estudo, também se analisou o impacto de todos os demais dados sociodemográficos, culturais e de saúde geral que, por sua vez, também poderiam influenciar a voz e a audição. A correlação entre prática de atividades físicas e qualidade de vida aqui identificada, já foi verificada anteriormente ${ }^{35}$. Apesar de evidências científicas da relação entre sedentarismo de distúrbio vocal ${ }^{36}$, o presente estudo não demonstrou associação entre desvantagem vocal e a prática de atividades físicas.

Embora a presente pesquisa não tenha encontrado evidências de relação entre o número de doenças crônicas e o impacto da audição no cotidiano, sua presença é apontada, na literatura, como um fator de risco para queixas auditivas em idosos ${ }^{7}$, incluindo-se a hipertensão e a diabetes mellitus $^{37}$. A relação dessas doenças com a qualidade de vida geral dos idosos, que foi verificada na amostra estudada, está de acordo com estudos revisados ${ }^{38,39}$, segundo os quais quanto maior o número de comorbidades, piores foram os escores de qualidade de vida e índices de felicidade.

O estudo apresenta, como limitações, o fato de não ter registrado se os sujeitos apresentavam queixas de voz e de audição, nem tampouco ter identificado, por meio de avaliação profissional específica, a presença dessas alterações, uma vez que o enfoque foi dado à funcionalidade, pela análise do impacto funcional de possíveis queixas de audição e/ou voz na vida dos sujeitos participantes do estudo.

Os resultados reforçam a importância de se avaliar, tanto clinicamente quanto no planejamento de políticas públicas, o impacto percebido pelo próprio idoso, considerando a influência dessa percepção na qualidade de $\operatorname{vida}^{7,12}$, para que o planejamento das ações seja baseado na real demanda do sujeito e da sociedade. Além disso, demonstram a necessidade de promoção de ações de autocuidado e empoderamento para os idosos ${ }^{40,41}$, já que se observa que, mesmo em idosos considerados ativos, há um impacto das modificações comunicativas nas atividades diárias, principalmente no que se refere às questões auditivas.

Aponta-se para a necessidade de mais estudos que explorem as mudanças senescentes na comunicação sob diferentes prismas, comparando os 
grupos de idosos com experiências e rotinas diversas; bem como as demandas destes sujeitos em relação a sua comunicação e sua percepção sobre a repercussão (ou não) das modificações desta ao longo do ciclo vital.

\section{Colaboradores}

JSC Chiossi participou da coleta e análise de dados e da redação do texto; BNG Goulart, FP Roque participaram da interpretação dos dados, da redação e revisão crítica do texto; e BM Chiari orientou todas as etapas desta pesquisa.

\section{Agradecimentos}

À Universidade Federal de São Paulo - UNIFESP, pelo apoio financeiro.

À coordenadora da UATI-UNIFESP, pelo apoio à realização da pesquisa. E aos idosos que participaram desta pesquisa pela disponibilidade e por consentir na divulgação dos dados. 


\section{Referências}

1. Instituto Brasileiro de Geografia e Estatística (IBGE). Sintese de indicadores sociais 2010. Rio de Janeiro: IBGE; 2010.

2. Organização Mundial de Saúde (OMS). Organização Pan-Americana da Saúde (OPAS). Envelhecimento ativo: uma politica de saúde. Brasília: OPAS; 2002.

3. Grimley-Evans J. Prevention of age-associated loss of autonomy: epidemiological approaches. J Chronic Dis 1984; 37(5):353-363.

4. Ramos LR. Fatores determinantes do envelhecimento saudável em idosos residentes em centro urbano: Projeto Epidoso, São Paulo. Cad Saude Publica 2003; 19(3):793-797.

5. Yorkston KM, Bourgeois MS, Baylor CR. Communication and aging. Phys Med Rehabil Clin N Am 2010; 21(2):309-319.

6. Cohen SM, Turley R. Coprevalence and impact of dysphonia and hearing loss in the elderly. Laryngoscope 2009; 119(9):1870-1873.

7. Gates GA, Mills JH. Presbycusis. Lancet 2005; 366(9491): 1111-1120.

8. Lejska M. Voice field measurements: a new method of examination: the influence of hearing on the human voice. J Voice 2004; 18(2):209-215.

9. Leeuw IMV, Mahieu HF. Vocal aging and the impact on daily life: a longitudinal study. J Voice 2004; 18(2):193202.

10. Geib LTC. Determinantes sociais da saúde do idoso. Cien Saude Colet 2012; 17(1):123-133.

11. Rosis ACA, Souza MRF, Iório MCM. Questionário Hearing Handicap Inventory for the Elderly - Screening: estudo da sensibilidade e especificidade. Rev Soc Bras Fonoaudiol 2009; 14(3):339-345.

12. Behlau M, Santos LMA, Oliveira G. Cross-Cultural Adaptation and Validation of the Voice Handicap Index Into Brazilian Portuguese. J Voice 2011; 25(3):354-359.

13. Power M, Quinn K, Schmidt S, WHOQOL-OLD Group. Development of the WHOQOL-Old module. Qual Life Res 2005; 14(10):2197-2214.

14. Moerman M, Martens JP, Dejonckere P. Application of the Voice Handicap Index in 45 patients with substitution voicing after total laryngectomy. Eur Arch Otorhinolaryngol 2004; 261(8):423-428.

15. Flerk MPA, Chachamovich E, Trentini CM. Projeto WHOQOL-OLD: método e resultados de grupos focais no Brasil. Rev Saude Publica 2003; 37(6):793-799.

16. Santiago LM, Novaes CO. Auto-avaliação da audição em idosos. Rev CEFAC 2009, 11(1):98-105.

17. Hidalgo JLT, Gras CB, Lapeira JT, Verdejo MAL, Campo JMC, Rabadán FE. Functional status of elderly people with hearing loss. Arch Gerontol Geriatr 2009; 49(1):88-92.

18. Sindhusake D, Mitchell P, Smith W, Golding M, Newall P, Hartley D, Rubin G. Validaton os self-reported hearing loss: the Blue Mountains hearing study. Int J Epidemiol 2001; 30(6):1371-1378

19. Barreto KML, Carvalho EMF, Falcão IV, Lessa FJD, Leite VMM. Perfil sócio-epidemiológico das mulheres idosas da Universidade Aberta à Terceira Idade no estado de Pernambuco. Rev Bras Saúde Matern Infant 2003; 3(3):339-354.
20. Irigaray TQ, Schneider RH. Prevalência de depressão em idosas participantes da Universidade para a Terceira Idade. Rev Psiquiatr RS 2007; 29(1):19-27.

21. Roque FP, Vinhas BR, Rebêlo FL, Guimarães HA, Araújo LZS, Goulart BNG, Chiari BM. Perfil socioeconômicocultural de uma universidade aberta à terceira idade: reflexo da realidade brasileira? Rev Bras Geriatr Gerontol 2011; 14(1):7-16.

22. Vuorialho A, Karienen P, Sorri M. Effect of hearing aids on hearing disability and quality of life in the elderly. Int J Audiol 2006; 45(7):400-405.

23. Boi R, Racca L, Cavallero A, Carpaneto V, Racca M, Dall'Acqua F, Ricchetti M, Santelli A, Odetti P. Hearing loss and depressive symptoms in elderly patients. Geriatr Gerontol Int 2012; 12(3):440-445.

24. Pugh KC, Crandell CC. Hearing loss, hearing handicap, and functional health status between African American and Caucasian American seniors. J Am Acad Audiol 2002; 13(9):493-502.

25. Dalton DS, Cruickshanks KJ, Klein BEK, Klein R, Wiley TL, Nondahl DM. The impact of hearing loss on quality of life in older adults. Gerontologist 2003; 43(5):661-668.

26. Gopinath B, Schneider J, Hickson L, McMahon CM, Burlutsky G, Leeder S, Mitchell P. Hearing handicap, rather than measured hearing impairment, predicts poorer quality of life over 10 years in older adults. Maturitas 2012; 72(2):146-151.

27. Golub SJ, Chen PH, Otto KJ, Hapner E, Johns III MM. Prevalence of perceived dysphonia in a geriatric population. JAGS 2006; 54(11):1736-1739.

28. Schneider S, Plank C, Eyshold U, Schützenberger A, Rosanowsky F. Voice function and voice-related quality of life in the elderly. Gerontology 2011; 57(2):109-114.

29. Kendall K. Presbyphonia: a review. Curr Opin Otolaryngol Head Neck Surg 2007; 15(3):137-140.

30. Costa HO, Matias C. O impacto da voz na qualidade da vida da mulher idosa. Rev Bras Otorrinolaringol 2005; 71(2):172-178.

31. Zraick RI, Risner BY, Smith-Olinde L, Greeg BA, Johnson FL, McWeeny EK. Patient versus partner perception of voice handicap. J Voice 2007; 21(4):485-494.

32. Plank C, Schneider S, Eysholdt U, Schützenberger A, Rosanowski F. Voice- and health-related quality of life in the elderly. J Voice 2011; 25(3):265-268.

33. Gampel D, Karsch UM, Ferreira LP. Percepção de voz e qualidade de vida em idosos professores e não professores. Cien Saude Colet 2010; 15(6):2907-2916.

34. Teixeira INDO, Neri AL. Envelhecimento bem-sucedido: uma meta no curso da vida. Psicol USP 2008; 19(1):81-94.

35. igueira HA, Figueira AA, Cader SA, Guimarães AC, Oliveira RJ, Figueira JA, Figueira OA, Dantas EH. Effects os a physical activity governmental health programme on the quality of life of elderly people. Scand J Public Health 2012; 40(5):418-422.

36. 3Assunção AA, Medeiros AM, Barreto SM, Gama ACC. Does regular practice of physical activity reduce the risk of dysphonia? Prev Med 2009; 49(6):487-489.

37. Mitchell P, Gopinath B, McMahont CM, Rochtchina E, Wang JJ, Boyages SC, Boyages SC, Leeder SR. Relationship of Type 2 diabetes to the prevalence, incidence and progression of age-related hearing loss. Diabet Med 2009; 26(5):483-488. 
38. Wikman A, Wardle J, Steptoe A. Quality of life and affective well-being in middle-aged and older people with chronic medical illnesses: a cross-sectional population based study. PLoS One [serial on the Internet] $2011 \mathrm{Apr}$ [cited 2012 Jan 15]; 6(4):e18952 [about 9 p.] Available from: http://www.1371/journal.pone.0018952.

39. Campolina AG, Dini PS, Ciconelli RM. Impacto de doenças crônicas na qualidade de vida de idosos da comunidade em São Paulo (SP, Brasil). Cien Saude Colet 2011; 16(6):2919-2925.

40. Carvalho SR. Os múltiplos sentidos da categoria "emporwerment" no projeto de promoção a saúde. Cad Saude Publica 2004; 20(4):1088-1095.

41. Melo MC, Souza AL, Leandro EL, Mauricio HA, Silva ID, Oliveira JMO. A educação em saúde como agente promotor de qualidade de vida para o idoso. Cien Saude Colet 2009; 14(Supl.1):1579-1586.

Artigo apresentado em 25/03/2013

Aprovado em 16/05/2013

Versão final apresentada em 23/05/2013 